\title{
Bem-estar e adaptabilidade de carreira na adaptação ao ensino superior
}

\author{
Bruno Rocha Almeidal \\ Maria Odilia Teixeiral \\ Faculdade de Psicologia da Universidade de Lisboa, Lisboa, Portugal
}

\section{Resumo}

No âmbito da adaptação ao ensino superior, é analisado o bem-estar subjetivo considerando adaptabilidade de carreira e outras variáveis vocacionais, académicas e sociais. Os participantes são universitários $(n=273)$ do $1^{\circ}$ e $2^{\circ}$ anos, em Escolas de Lisboa, sendo 82\% mulheres, que responderam às Escalas de Adaptabilidade de Carreira, de Desenvolvimento e Bem-estar, e Questionário de Ajustamento e Adaptação ao Ensino Superior. Os resultados mostram como preditores de Bem-estar a Adaptabilidade de Carreira, designadamente Controlo e Confiança e fatores adaptativos de Perspetiva de Carreira, Gestão Tempo, Relação com Pares, sendo Ansiedade à Avaliação fator negativo. A estatística inferencial, evidencia índices de maior adaptação dos estudantes do $2^{\circ}$ ano, comparativamente aos do $1^{\circ}$ ano. São discutidas implicações nas intervenções.

Palavras-chave: Ajustamento escolar, bem-estar subjetivo, adaptabilidade de carreira, fatores psicossociais, ensino superior

Abstract: Well-being and career adaptability in adaptation to college

In the context of adaptation to higher education, subjective well-being is analyzed, considering career adaptability and other vocational, academic and social variables. The participants are first- and second-year undergraduates ( $n=273$ ), from Lisbon schools, among which $82 \%$ are female. They responded to the Career Adaptability Scale, Development and Well-being Scale and the Adjustment and Adaptation to Higher Learning Questionnaire. The results show that Career Adaptability, namely Control and Confidence, and the adaptive factors of Career Perspective, Time Management, Relationship with Peers, and Evaluation Anxiety as a negative factor, are predictors of well-being. Furthermore, inferential statistics shows greater adaptation in second year students in comparison with first year students. Implications for interventions are discussed.

Keywords: School adjustment, subjective well-being, career adaptability, psychosocial factors, higher education

\section{Resumen: Bienestar y adaptabilidad de carrera en la adaptación a la educación superior}

En el ámbito de la adaptación a la enseñanza superior, se analiza el bienestar subjetivo considerando la adaptabilidad de carrera y otras variables vocacionales, académicas y sociales. Los participantes son universitarios $(n=273)$ de primer y segundo año de universidades de Lisboa ( $82 \%$ mujeres), que respondieron las Escalas de Adaptabilidad de Carrera, de Desarrollo y de Bienestar; y el Cuestionario de Ajuste y Adaptación a la Educación Superior. Los resultados mostraron, como predictores del Bienestar, la Adaptabilidad de Carrera, específicamente el Control y la Confianza, y factores adaptativos de Perspectiva de Carrera, Gestión del Tiempo, Relación con Iguales, siendo la ansiedad ante las evaluaciones un factor negativo. La estadística inferencial evidencia índices de mayor adaptación en los estudiantes de segundo año con relación a los de primer año. Son analizadas las implicaciones de las intervenciones.

Palabras clave: Adaptación escolar, bienestar subjetivo, adaptabilidad de carrera, factores psicosociales, educación superior

\footnotetext{
${ }^{1}$ Endereço para correspondência: Alameda da Universidade, 1649-013, Lisboa, Portugal. Fone: 2179345 54. E-mails: bmra.18@gmail.com; moteixeira@psicologia.ulisboa.pt
} 
Em Portugal, as questões da transição, adaptação e sucesso no ensino superior são entendidas no contexto de políticas nacionais e europeias, num quadro multidimensional de variáveis pessoais, sociodemográficas, académicas, vocacionais e institucionais (e.g., Soares, Pinheiro, \& Canavarro, 2015). A temática do ensino superior abrange as próprias caraterísticas do mundo contemporâneo no contexto de globalização e flexibilidade. Isto exige dos estudantes um conjunto de competências transversais, consideradas como elementos críticos no atual mercado de trabalho, associadas às competências funcionais do conhecimento (Almeida, Araújo, \& Martins, 2016; Briggs, Clark, \& Hall, 2012). Neste sentido, as instituições de ensino superior reúnem propósitos de natureza académica aos conhecimentos culturais, artísticos, tecnológicos, numa relação de proximidade com a sociedade e de facilitação do acesso igualitário à aprendizagem ao longo da vida e mobilidade social (Soares et al., 2015).

Nas últimas décadas, a Declaração de Sorbonne em 1998 e a Declaração de Bolonha em 1999, aliadas ao processo de democratização do ensino, transformaram o ensino superior português (Soares et al., 2015). No acompanhamento destas mudanças, foram criadas estruturas de apoio aos estudantes, principalmente dirigidas ao primeiro ano curricular, com propósitos de facilitar a adaptação e integração (Almeida, et al., 2016; Soares et al., 2015). No entanto, os indicadores da Direção-Geral de Estatísticas da Educação e Ciência (DGEEC, 2015) mostram que no $1^{\circ}$ ano de licenciatura os abandonos são cerca de $12 \%$ e as mudanças de curso rondam os $9,7 \%$, ou seja, cerca de 1 em cada 5 estudantes não se encontra no curso em que se inscreveu no ano letivo de 2012/2013, seja por abandono ou mudança. Esta situação exige reflexão e identificação dos fatores que facilitam a transição e consequentemente o melhoramento do sucesso académico, com irrevogáveis efeitos positivos para estudantes, famílias e instituições.

\section{Transições, carreira e adaptabilidade}

A transição para o ensino superior é, para muitos estudantes, a realização de um sonho, mas pode conter obstáculos que comprometem o bem-estar e sucesso (Almeida et al., 2016; Chickering \& Schlossberg, 1995; Costabile, Cornoldi, De Beni, Manfredi, \& Figliuzzi, 2013; Ferreira \& Fernandes, 2015; Soares, et al., 2015). As transições são próprias do desenvolvimento ao longo da vida, com fases e tarefas específicas, com características transformadoras e criadoras (Chickering \& Schlossberg, 1995; Savickas, 2013). As transições envolvem desafios, reavaliações e ações, alinhados com objetivos individuais de sucesso e pressupondo adaptação entre o indivíduo e o meio ao longo do processo. Enquanto processos psicológicos, as transições são alicerçadas na perceção e expressão de mudanças (Chickering \& Schlossberg, 1995; Soares, et al., 2015), que envolvem papéis, atitudes e experiências. Entre as várias variáveis que concorrem para o sucesso, a literatura realça o papel dos fatores de carreira como componente crítica da adaptação, satisfação e desempenho no ensino superior (e.g., Duffy, Douglass, \& Autin, 2015; Savickas, 2013; Teixeira \& Costa, 2017).

De um ponto de vista socioconstrutivista, a carreira é um processo ao longo da vida, abrangente e flexível, envolvendo atividades, experiências, tempos livres, redes interpessoais, saúde mental, personalidade e temas de vida, bem como a concomitância e (des)vinculação de papéis (Chickering \& Schlossberg, 1995; Savickas, 2013). Ainda nesta linha, importa salientar a proposta de Lent e Brown (2013), cujo modelo sociocognitivo de autogestão da carreira foca prioritariamente os processos de transição, com integração dos fatores pessoais, fatores contextuais, mediadores cognitivos e competências de adaptabilidade da carreira.

A adaptabilidade de carreira corresponde a um construto psicossocial, definido como a prontidão para lidar com tarefas previsíveis na carreira e ajustamentos imprevisíveis decorrentes de mudanças, recorrendo a atitudes de planeamento, exploração pessoal e ambiental e tomada de decisão informada e adaptada, orientando o indivíduo para transições futuras de uma forma realista e preparada (Savickas, 2013; Savickas \& Porfeli, 2012). Este construto tem natureza multidimensional e hierárquica, sendo dimensão de primeira ordem um indicador geral de adaptabilidade, que agrega fatores de segunda ordem definidos como preocupação, controlo, confiança e curiosidade. A Preocupação é relativa à esperança e otimismo na orientação para o futuro; é uma atitude de antecipação relacionada com carreira, e preparação para tarefas, mudanças e compromissos (Duarte et al., 2015; Rudolph, Lavigne, Katz, \& Zacher, 2017). A preocupação ajuda a pessoa a preparar o que deseja para o seu futuro (Savickas \& Porfeli, 2012). O Controlo expressa a influência que a pessoa perceciona sobre si e o meio; significa poder e responsabilidade pela autogestão e gestão dos contextos. Tem uma componente de autodeterminação, manifestando-se como positividade, tomada de decisão, responsabilidade, eficácia e esforço com objetivos adaptativos (Duarte et al., 2015; Rudolph et al., 2017; Savickas \& Porfeli, 2012). O controlo permite que os indivíduos se tornem responsáveis por moldarem-se a si mesmos e aos ambientes para encontrar o que vem a seguir, usando autodisciplina, esforço e persistência (Savickas \& Porfeli, 2012, p. 662). A Confiança representa a capacidade de manter objetivos e metas apesar de 
obstáculos; reflete autossegurança, crença nas capacidades para atingir objetivos de carreira; relaciona-se com maior eficiência e cuidado em concretizar bem tarefas, e desejo de aprender novas competências e demonstrar habilidade (Duarte et al., 2015; Rudolph et al., 2017); traduz o conceito de crença de autoeficácia (Bandura, 1997) para o ambiente de carreira (Lent \& Brown, 2013). A Curiosidade representa uma dimensão referente aos comportamentos de exploração vocacional e envolvimento em atividades de carreira. Diz respeito à exploração e reflexão sobre possibilidades pessoais e de carreira (Duarte et al., 2015; Rudolph et al., 2017; Savickas \& Porfeli, 2012).

Estas dimensões da adaptabilidade da carreira correspondem a estratégias autorregulatórias que podem ser mobilizadas para lidar com transições e tarefas no desenvolvimento da carreira (Duarte et al., 2015; Rudolph et al., 2017; Savickas \& Porfeli, 2012). Enquanto competências, influenciam outras dimensões (e.g., envolvimento, satisfação, ansiedade, transições, stress) e resultados no âmbito da carreira, nomeadamente nas áreas escolares e profissionais (Maggiori, Johnston, Krings, Massoudi, \& Rossier, 2013).

\section{Bem-estar}

O bem-estar representa o funcionamento positivo, considerando emoções positivas, envolvimento, vida com sentido, relações positivas e realizações (Seligman, 2011). Nas últimas décadas, o conceito de bem-estar tem sido cada vez mais relevante na literatura (e.g., Keyes, 2006; Minkov, 2009; Robertson, 2015; Teixeira \& Costa, 2017) culminando em estudos de bem-estar psicológico e bem-estar subjetivo. A aceção de bem-estar psicológico está mais ligada ao desenvolvimento do adulto e saúde mental, enquanto o bem-estar subjetivo está mais associado a aspetos psicossociais, sentimentos de felicidade e satisfação, às condições e circunstâncias da vida (Diener \& Ryan, 2009; Siqueira \& Padovam, 2008). O conceito de bem-estar subjetivo tende a agregar a componente cognitiva de avaliação da vida, em termos de satisfação e emoções positivas e a componente hedonística da felicidade e do prazer (Minkov, 2009). Diener et al. (2009) incluem aspetos de ambos os conceitos de bem-estar numa medida que avalia florescimento. $\mathrm{O}$ conceito de florescimento (flourishing) foi proposto por Keyes (2006), referindo um estado de procura de emoções positivas e desenvolvimento do potencial humano, nas vertentes sociais e psicológicas. Este conceito está na génese dos padrões de qualidade de vida individual e coletiva (Seligman, 2011), e é significativo no mundo académico e de carreira. A investigação sobre o bem-estar subjetivo tem vindo a identificar variáveis associadas às avaliações que as pessoas fazem da vida, com base nas experiências de prazer e felicidade, que refletem cognições relativas à satisfação com a vida, experiências emocionais (negativas e positivas) e felicidade (Diener \& Ryan, 2009). Bem-estar também está associado a variáveis culturais, económicas e sociais (Minkov, 2009).

\section{Bem-estar e Carreira}

O tema do bem-estar faz a ponte entre Psicologia Positiva e a Psicologia da Carreira, pois nestas duas perspetivas psicológicas é atribuído um papel chave ao funcionamento positivo, ao desenvolvimento das potencialidades e à procura de realização pessoal e social (Lent \& Brown, 2013; Robertson, 2015; Teixeira \& Costa, 2017). A literatura demonstra o significado do bem-estar na carreira, pelas relações observadas entre bem-estar e produtividade, satisfação na carreira, satisfação com a vida, envolvimento académico (e.g., Keyes, 2006; Merino-Tejedor, Hontangas, \& Boada-Grau, 2016; Teixeira \& Costa, 2017). Geralmente, uma perceção positiva de bem-estar e satisfação com a vida melhoram significativamente as áreas da saúde e longevidade, trabalho, rendimento e relações sociais (Diener \& Ryan, 2009); a satisfação nestas áreas de vida também influencia o bem-estar geral. Neste sentido, podemos falar de bem-estar geral ou bem-estar aplicado a contextos específicos. A adaptação com sucesso pode levar a experiências de bem-estar noutros domínios (Rudolph et al., 2017). A literatura tem salientado a natureza recíproca da relação de bem-estar com a satisfação na carreira, e de bem-estar com a perceção de congruência entre caraterísticas pessoais e de curso (e.g., Minkov, 2009; Teixeira \& Costa, 2017). Assim, é imperativo identificar as fontes que contribuem tanto para o sentimento geral positivo como para potenciar os recursos de carreira, já que o bem-estar é definido como um dos resultados mais preditivos da adaptação e as variáveis de carreira como fatores preditivos de bem-estar (e.g., Rudolph et al., 2017; Teixeira \& Costa, 2017).

Entre as variáveis do domínio da carreira, é relevante estudar a ligação de bem-estar com a adaptabilidade de carreira, uma vez que os recursos autorregulatórios da carreira potenciam o desenvolvimento e o sucesso na adaptação a contextos de carreira. A investigação identifica a relação entre adaptabilidade e bem-estar em vários contextos, ao nível da satisfação com a carreira, trabalho e escola, empregabilidade, desempenho, envolvimento, compromisso, identidade de carreira, comportamentos de saúde, burnout, stress, ansiedade, satisfação com a vida e afetos positivos (Duffy et al., 2015; Hirschi, 2009; Konstam, Celen-Demirtas, Tomek, \& Sweeney, 2015; Maggiori 
et al., 2013; Merino-Tejedor et al., 2016; Rudolph et al., 2017; Teixeira \& Costa, 2017). Recentemente, o Modelo de Autogestão da Carreira de Lent e Brown (2013) propõe integrar diferentes conceções de adaptabilidade da carreira, em que são incluídas as dimensões propostas no modelo da Savickas (2013) com os processos cognitivos de adaptação da carreira, em que entram traços de personalidade e mecanismos cognitivos como autoeficácia.

\section{Vivências académicas}

Os processos de adaptação são perspetivados ao nível individual e nível institucional, exigindo um foco na interação entre indivíduo e instituição na análise do bem-estar, aprendizagem e desenvolvimento da carreira (Lent \& Brown, 2013; Soares et al, 2015).

Nas vivências no ensino superior, os modelos de impacto de Astin (e.g., Astin \& Sax, 1998) e de Pascarella (e.g., Pascarella \& Terenzini, 1980) consideram o envolvimento do estudante como uma das componentes mais importantes, realçando assim o papel da agência pessoal, suporte social e institucional como grandes contribuidores no processo de transição e adaptação e à probabilidade de sucesso nos mesmos (e.g., Ferreira \& Fernandes, 2015; Lent, Taveira, Sheu, \& Singley, 2009; Soares et al., 2015). Estes modelos enfatizam contextos dentro e fora da sala de aula, em termos de atividades (extra)curriculares e a interação com os espaços, onde a criação de espaços e pedagogias que promovam cooperação, responsabilidade, desenvolvimento, confiança, metacognição, entre outros, são essenciais. Tomam-se como exemplo programas ou unidades curriculares que desenvolvam competências e promovam adaptação, e atividades de voluntariado. Além dos fatores institucionais, o suporte social, principalmente nas relações com pares, é fonte com grande impacto no desenvolvimento, em termos de identidade, aprendizagem e valores, bem-estar, satisfação, saúde física e psicológica, ajustamento e adaptação (Bandura, 1997; Diener \& Ryan, 2009; Lent, et al., 2009; Ryff \& Keyes, 1995; Teixeira \& Costa, 2017).

Num quadro teórico que perspetiva a adaptação ao ensino superior no âmbito da carreira e do contexto académico, é objetivo desta investigação analisar o bem-estar subjetivo considerando adaptabilidade de carreira, perceção das questões de carreira no âmbito do próprio curso e variáveis de índole social e da aprendizagem no ambiente de vivência académica. As hipóteses em estudo seguem as tendências da literatura:

- Hipótese 1 - níveis elevados de adaptabilidade da carreira influenciam positivamente o bem-estar;
- Hipótese 2 - perspetiva positiva de carreira face ao curso influencia o bem-estar;

- Hipótese 3 - relação entre pares influencia positivamente o bem-estar;

- Hipótese 4 - fatores académicos influenciam o bem-estar;

- Hipótese 5 - os estudantes do $2^{\circ}$ ano revelam níveis de adaptação superiores aos do $1^{\circ}$ ano.

\section{Método}

\section{Participantes}

A amostra é constituída por 273 estudantes que frequentam o $1^{\circ}(57,1 \%)$ e $2^{\circ}$ anos $(42.9 \%)$ do ensino superior, de diferentes Escolas da cidade de Lisboa, em áreas de Ciências (12\%), Saúde (9\%), Tecnologias (7\%), Agricultura e Recursos Naturais (1\%), Arquitetura, Artes Plásticas e Design (2\%), Ciências da Educação e Formação de Professores (9\%), Direito, Ciências Sociais e Serviços (54\%), Economia, Gestão e Contabilidade (3\%), Secretariado e Tradução (3\%), Educação Física, Desporto e Artes do Espetáculo (2\%). Os participantes têm idades compreendidas entre 18 e 24 anos $(M=19, D P=1)$, sendo cerca de $82 \%$ do sexo feminino.

\section{Instrumentos}

\section{Questionário sociodemográfico}

Este questionário inclui variáveis sociodemográficas sobre sexo, idade, ano curricular $\left(1^{\circ}\right.$ ou $\left.2^{\circ}\right)$.

\section{Escala de Adaptabilidade de Carreira (Career Adapt-} Abilities Scale; Savickas et al., 2011)

É um instrumento de autorrelato, cuja construção decorreu no projeto Life Design, com participação de investigadores de 13 países, num grupo liderado por Mark Savickas. Avalia as quatro dimensões associadas ao construto de adaptabilidade: preocupação, controlo, curiosidade e confiança. A versão portuguesa (Duarte et al., 2012) contém 28 itens numa escala de Likert de 5 pontos (1 - Muito pouco; 5 - Muito) e em formato de afirmação, que estão distribuídos pelas quatro escalas, com 7 itens cada.

Reporta-se que o estudo de Duarte et al. (2012) com uma amostra do $9^{\circ}$ ao 12 , adultos empregados (com formação superior) e desempregados, demonstra precisão favorável nos coeficientes alfa, sendo na escala total .90, e nas subescalas Preocupação, Controlo, Curiosidade e Confiança $-.76, .69, .78$ e .79 , respetivamente. Realça-se também o 
estudo de Teixeira, Bardagi, Lassance, Magalhães e Duarte (2012) efetuado no Brasil com uma versão de 22 itens, numa amostra de adultos, em que a maioria tinha formação superior e estava empregado. Neste estudo, o coeficiente alfa para a escala total é .91, e nas subescalas Preocupação, Controlo, Curiosidade e Confiança são $.77, .76, .81$ e .82 , respetivamente (Teixeira et al., 2012).

\section{Escala de Desenvolvimento e Bem-estar (Flourishing} Scale; Diener et al., 2009)

É um instrumento de autorrelato cujo conteúdo representa aspetos do funcionamento positivo como relações interpessoais, sentimentos de competência, significado e propósito na vida. É constituído por 8 itens, em forma de afirmação, numa escala de Likert de 7 pontos (1 - Discordo fortemente a 7 - Concordo fortemente). Trata-se de uma escala unidimensional, onde os itens convergem na avaliação do construto global de bem-estar. Valores elevados indicam uma autoavaliação muito positiva em diversas áreas de funcionamento, além de recursos e forças psicológicas, oferecendo uma visão geral dos domínios considerados significativos, apesar de não avaliar diretamente as facetas do bem-estar psicológico (Diener et al., 2009).

Em diferentes amostras, os estudos têm vindo a confirmar a validade e precisão da medida, nomeadamente a natureza unifatorial e a consistência interna, cujos coeficientes alfa se situam em .87 e de teste-reteste em .71 (Diener et al., 2009). Numa amostra portuguesa do ensino superior, os indicadores confirmam as propriedades psicométricas desta medida, com coeficiente alfa de .88 (Teixeira \& Costa, 2017).

Questionário de Ajustamento e Adaptação ao Ensino Superior (QAAES; Teixeira \& Costa, 2017).

É um instrumento de autorrelato, cuja versão utilizada neste estudo inclui 40 itens, revistos com base em questionários anteriores sobre transição e adaptação no ensino superior (e.g., Soares, Almeida, \& Ferreira, 2006), especialmente o questionário elaborado por Teixeira e Costa (2017).

Os resultados dos itens desta nova versão foram submetidos à análise em componentes principais, sendo impostos cinco componentes com base no teste da escarpa, que explicam cerca de 53\% da variância total. Após rotação varimax, os cinco componentes foram interpretados com base na consistência dos seus conteúdos como correspondendo às dimensões Ansiedade à Avaliação (12 itens), Relação com Pares (9 itens), Gestão do Tempo (6 itens), Perspetiva de Carreira (7 itens) e uma componente designada de Estudo e Aprendizagem (5 itens), cujos conteúdos agregam itens da relação com professores, acompanhamento dos conteúdos, resultados escolares e métodos de estudo. Os itens são em formato de afirmação, com resposta em escala de Likert de 5 pontos.

\section{Procedimentos}

Éticos e deontológicos

O Requerimento de Aprovação de Projeto de Investigação foi submetido e aprovado pela Comissão Especializada do Conselho Científico da Faculdade de Psicologia da Universidade de Lisboa. O consentimento informado é disponibilizado previamente ao preenchimento do questionário.

\section{Recolha de dados}

A aplicação dos instrumentos recorreu da técnica de bola de neve em formato online, com distribuição através de redes sociais e correio eletrónico para várias faculdades. Foi feita ainda a aplicação em formato papel, com preenchimento em aulas. Trata-se de uma amostra de conveniência. Os questionários apresentavam-se na seguinte ordem: 1. Questionário sociodemográfico; 2. Escala de Adaptabilidade de Carreira; 3. Escala de Desenvolvimento e Bem-estar; e 4. Questionário de Ajustamento e Adaptação ao Ensino Superior.

\section{Análise de dados}

Nas análises estatísticas, procedeu-se primeiramente à estimativa das características das distribuições dos resultados e do grau de consistência interna das diferentes medidas, pelo coeficiente alfa de Cronbach. Em segundo lugar, foram usados procedimentos de estatística inferencial para avaliar o grau de associação das variáveis (correlações) e o teste $t$ de Student para comparação de médias entre grupos. De seguida, efetuou-se uma regressão linear simples e múltipla para analisar relações de causalidade e identificar variáveis preditoras de bem-estar da amostra. Os dados foram tratados com o software estatístico IBM SPSS Statistics 24.

\section{Resultados}

\section{Análise dos instrumentos}

\section{Medidas de tendência central e de dispersão}

$\mathrm{Na}$ análise das distribuições, as medidas de tendência central e de dispersão (tabela 1) apresentam, no geral, 
variabilidade favorável dos resultados em todos os instrumentos. De notar os valores superiores à média teórica na maioria das escalas, revelando alguma assimetria. No entanto, mantém-se o pressuposto de distribuição normal segundo o teorema do limite central.

Nesta amostra, a hierarquia das médias relativas à Adaptabilidade de Carreira permite observar resultados elevados em Controlo e Confiança, sendo a média mais baixa em Curiosidade. Os estudantes da amostra tendem a expressar níveis elevados de Desenvolvimento e Bem-estar. Não é avaliada a hierarquia das médias do Questionário de Ajustamento e Adaptação ao Ensino Superior pelo número desigual de itens nas subescalas.

\section{Precisão}

Na tabela 1 incluem-se também os coeficientes alfa para as medidas usadas.

Na Escala de Adaptabilidade de Carreira o coeficiente alfa para a escala total situa-se em .92 , e nas subescalas Preocupação em .80, Controlo em .78, Curiosidade em.81 e Confiança em .88. Estes indicadores de precisão são semelhantes aos dos estudos anteriores (e.g., Duarte et al., 2012; Savickas \& Porfeli, 2012).

Na Escala de Desenvolvimento e Bem-estar o coeficiente alfa é de .87 , semelhante a estudos anteriores (e.g., Diener et al., 2009; Teixeira \& Costa, 2017).

No Questionário de Ajustamento e Adaptação ao Ensino Superior observam-se coeficientes alfa de .92 para a escala de Ansiedade à Avaliação, .88 para Relação com Pares, .79 para Gestão de Tempo, .79 para Perspetiva de Carreira e .66 para Estudo e Aprendizagem.

\section{Correlações entre os resultados das escalas}

$\mathrm{Na}$ tabela 2 observam-se correlações entre |.04| (Ansiedade à Avaliação e Relação com Pares) e |.62| (Controlo e Confiança), com correlações significativas entre os resultados da maioria dos instrumentos. Salientam-se correlações superiores a $|.30|$, seguindo o critério de que representa uma correlação de força média (Steele, Andrews, \& Upton, 2012).

Observam-se resultados positivos e significativos entre as subescalas de Adaptabilidade de Carreira, variando entre .39 (Preocupação e Controlo) e .62 (Controlo e Confiança). Os resultados da Escala de Desenvolvimento e Bem-estar associam-se aos das subescalas de Adaptabilidade de Carreira, Relação com Pares, Perspetiva de Carreira e Gestão de Tempo, e ainda à Ansiedade à Avaliação, sendo esta negativa. Além das referidas, a subescala de Perspetiva de Carreira tem associações positivas com Preocupação, Controlo e Confiança. De referir ainda as associações positivas entre a subescala Estudo e Aprendizagem com Gestão do Tempo e com Confiança.

\section{Diferenças entre grupos}

$\mathrm{Na}$ análise dos resultados pelo ano curricular, os dados do teste $t$ de Student indicam superioridade das médias do grupo do $2^{\circ}$ ano nas escalas Controlo $(p=.007)$, Curiosidade $(p=.007)$, Confiança $(p=.004)$ e de Estudo e Aprendizagem $(p=.015)$. O grupo do $1^{\circ}$ ano expressa uma média significativamente superior na escala Ansiedade à Avaliação $(p=.006)$. $\mathrm{O}$ teste de Levene indica homogeneidade das variâncias em todas as escalas. Na classificação de Cohen, o tamanho do efeito é médio para Controlo $(d$ de Cohen $=.34)$ Curiosidade $(d$ de Cohen $=.34)$, Confiança $(d$ de Cohen $=.36)$, Ansiedade à Avaliação $(d$ de Cohen $=.36)$ e Estudo e Aprendizagem $(d$ de Cohen $=.31)$.

Tabela 1

Estatística descritiva dos instrumentos $(n=273)$

\begin{tabular}{lccccccc}
\hline \multicolumn{1}{c}{ Escalas } & $\mathrm{M}$ & $\mathrm{DP}$ & $\mathrm{Sk}$ & $\mathrm{Ku}$ & $\mathrm{Min}$ & $\mathrm{Max}$ & alfa de Cronbach \\
\hline Adaptabilidade de Carreira & 106.50 & 13.28 & 0.00 & 0.25 & 59 & 140 & .92 \\
Preocupação & 26.26 & 4.14 & -0.09 & -0.03 & 14 & 35 & .80 \\
Controlo & 27.16 & 3.99 & -0.37 & 0.07 & 15 & 35 & .78 \\
Curiosidade & 25.93 & 4.02 & 0.13 & -0.29 & 15 & 35 & .81 \\
Confiança & 27.14 & 4.38 & -0.55 & 1.36 & 7 & 35 & .88 \\
Desenvolvimento e Bem-estar & 41.99 & 7.38 & -0.98 & 2.03 & 11 & 56 & .87 \\
Ansiedade à Avaliação & 39.15 & 10.8 & 0.01 & -0.72 & 14 & 60 & .92 \\
Relação com Pares & 34.13 & 6.95 & -0.55 & -0.13 & 13 & 45 & .88 \\
Perspetiva de Carreira & 25.86 & 4.63 & -0.44 & 0.56 & 9 & 35 & .79 \\
Gestão de Tempo & 19.30 & 4.28 & -0.14 & -0.23 & 8 & 30 & .79 \\
Estudo e Aprendizagem & 16.79 & 2.89 & -0.18 & 0.21 & 9 & 25 & .66 \\
\hline
\end{tabular}


Tabela 2

Correlações de Pearson entre os resultados de todas as escalas

\begin{tabular}{ccccccccccc}
\hline & $\mathrm{P}$ & $\mathrm{Ct}$ & $\mathrm{Cr}$ & $\mathrm{Cf}$ & $\mathrm{DBE}$ & $\mathrm{AA}$ & $\mathrm{RP}$ & $\mathrm{PC}$ & $\mathrm{GT}$ & EA \\
\hline $\mathrm{P}$ & 1 & $.39^{* *}$ & $.44^{* *}$ & $.55^{* *}$ & $.43^{* *}$ & -.09 & .06 & $.39^{* *}$ & $.20^{* *}$ & $.27^{* *}$ \\
$\mathrm{Ct}$ & & 1 & $.51^{* *}$ & $.62^{* *}$ & $.59^{*}$ & $-.34^{* *}$ & $.27^{* *}$ & $.36^{* *}$ & $.27^{* *}$ & $.29^{* *}$ \\
$\mathrm{Cr}$ & & & 1 & $.61^{* *}$ & $.39^{* *}$ & $-.14^{*}$ & $.17^{* *}$ & $.21^{* *}$ & $.24^{* *}$ & $.25^{* *}$ \\
$\mathrm{Cf}$ & & & & 1 & $.54^{* *}$ & $-.27^{* *}$ & $.18^{* *}$ & $.36^{* *}$ & $.29^{* *}$ & $.36^{* *}$ \\
$\mathrm{DBE}$ & & & & & 1 & $-.33^{* *}$ & $.36^{* *}$ & $.48^{* *}$ & $.37^{* *}$ & $.24^{* *}$ \\
$\mathrm{AA}$ & & & & & & 1 & -.04 & $-.18^{* *}$ & $-.23^{* *}$ & $-.27^{* *}$ \\
$\mathrm{RP}$ & & & & & & & 1 & $.16^{*}$ & $.13^{*}$ & .11 \\
$\mathrm{PC}$ & & & & & & & & 1 & $.15^{*}$ & $.23^{* *}$ \\
$\mathrm{GT}$ & & & & & & & & & 1 & $.51^{* *}$ \\
$\mathrm{EA}$ & & & & & & & & & & 1 \\
\hline
\end{tabular}

Nota: P - Preocupação; Ct - Controlo; Cr - Curiosidade; Cf - Confiança; DBE - Desenvolvimento e Bem-estar; AA - Ansiedade à Avaliação; RP - Relação com Pares; PC - Perspetiva de Carreira; GT - Gestão de Tempo; EA - Estudo e Aprendizagem

** Correlação significativa a .01; * correlação significativa a .05

Tabela 3

Diferenças entre médias pela variável ano curricular (Teste-t)

\begin{tabular}{|c|c|c|c|c|c|c|c|}
\hline \multirow{2}{*}{ Escalas } & \multicolumn{2}{|c|}{$1^{\circ}$ ano $(n=150)$} & \multicolumn{2}{|c|}{$2^{\circ}$ ano $(n=113)$} & \multirow{2}{*}{$F$} & \multirow{2}{*}{$t$} & \multirow{2}{*}{$p$} \\
\hline & $\mathrm{M}$ & DP & M & DP & & & \\
\hline Controlo & 26.57 & 3.89 & 27.92 & 4.00 & 1.29 & -2.74 & .007 \\
\hline Curiosidade & 25.35 & 3.89 & 26.70 & 4.07 & 0.51 & -2.73 & .007 \\
\hline Confiança & 26.47 & 4.55 & 28.03 & 3.99 & 0.89 & -2.89 & .004 \\
\hline Ansiedade à Avaliação & 40.77 & 10.83 & 36.97 & 10.48 & 0.23 & 2.80 & .006 \\
\hline Estudo e Aprendizagem & 16.41 & 2.69 & 17.29 & 3.07 & 1.54 & -2.44 & .015 \\
\hline
\end{tabular}

\section{Regressão linear}

Considerando a variável dependente bem-estar como componente de adaptação ao ensino superior, procedeu-se à estimativa de modelos de regressão linear simples, incluindo como variável independente a Escala de Adaptabilidade (resultados totais). O modelo de regressão linear simples é estatisticamente significativo, explicando $41 \%$ da variância $\left[F(1,250)=176.14 ; R^{2} a=.41\right.$; $p=.000]$. Nestes dados, foram identificados e retirados seis casos outliers a três desvios-padrão. O valor de DurbinWatson é 1,83, assegurando a independência dos erros.

Em seguida, para obter um modelo mais parcimonioso explicativo do Bem-estar, consideraram-se as variáveis independentes Preocupação, Controlo, Curiosidade, Confiança, Ansiedade à Avaliação, Relação com Pares, Perspetiva de Carreira, Gestão do Tempo e Estudo e Aprendizagem. Foram testados os pressupostos do modelo, nomeadamente o da distribuição normal, multicolinearidade, homogeneidade e independência dos erros, expressando um valor de Durbin-Watson de 1.95. Através dos resultados decorrentes da distância de Mahalanobis identificaram-se quatro outliers com potencial impacto na estimativa da reta de regressão, sendo o modelo de regressão calculado sem estes valores extremos. Consideram-se efeitos significativos a partir de $p<.05$.

$\mathrm{O}$ modelo estimado pelo método stepwise é estatisticamente significativo, explicando $60 \%$ da variância $\left[F(6,234)=59.67 ; R^{2} a=.60 ; p=.000\right]$. Neste modelo, as variáveis independentes são agrupadas em seis blocos, expressando um modelo final (tabela 4) com seis variáveis, que por ordem de entrada são Controlo $(\beta=.30, t(234)$ $=5.37 ; p<.01)$, Perspetiva de Carreira $(\beta=.26, t(234)=$ 5.64; $p<.01)$, Gestão do Tempo $(\beta=.17, t(234)=3.89$; $p<.01$ ), Ansiedade à Avaliação, com relação negativa $(\beta=-.17, t(234)=-3.830 ; p<.01)$, Relação com Pares $(\beta=.15, t(234)=3.48 ; p<.01)$ e Confiança $(\beta=.17$, $t(234)=3.04 ; p<.01)$. O valor de VIF varia entre 1.09 e 1.87 . 
Tabela 4

Estatística da regressão linear múltipla por stepwise-Bem-estar

\begin{tabular}{|c|c|c|c|}
\hline Preditores & $\beta$ & $\mathrm{t}$ & $\mathrm{p}$ \\
\hline Controlo & .30 & 5.37 & .000 \\
\hline Perspetiva de Carreira & .26 & 5.64 & .000 \\
\hline Gestão do Tempo & .17 & 3.89 & .000 \\
\hline Ansiedade à Avaliação & -.17 & -3.83 & .000 \\
\hline Relação com Pares & .15 & 3.48 & .001 \\
\hline Confiança & .17 & 3.04 & .003 \\
\hline
\end{tabular}

\section{Discussão}

$\mathrm{Na}$ temática da adaptação ao ensino superior, este estudo analisa o bem-estar dos estudantes de $1^{\circ}$ e $2^{\circ}$ ano, pelo contributo das variáveis de carreira (Adaptabilidade e Perspetiva de Carreira), variáveis sociais (Relação com Pares) e académicas (Gestão do Tempo, Ansiedade à Avaliação e Estudo e Aprendizagem). A discussão dos resultados segue os objetivos e hipóteses estabelecidas no âmbito da literatura sobre carreira, transições e bem-estar, considerando limitações à generalização dos resultados pela natureza da amostra e design de investigação adotado. De salientar que nos dados emergem indicadores favoráveis à qualidade metrológica dos instrumentos, designadamente na consistência interna das medidas.

Os resultados da regressão linear simples e múltipla confirmam a influência da Adaptabilidade de Carreira, designadamente nas dimensões Controlo e Confiança, no bem-estar dos estudantes desta amostra. Estes dados confirmam parcialmente a $\mathrm{H} 1$ e são corroborados pela literatura (e.g., Rudolph et al., 2017). Controlo foi o recurso mais consistente e menos influenciado pela presença de outras variáveis, sugerindo ser a mais relevante para explicar bem-estar neste contexto e amostra, também visível na correlação positiva e significativa (.59) entre Controlo e Bem-estar. Estes resultados são ainda confirmados por trabalhos anteriores que destacam o Controlo como mecanismo interno que opera no sucesso pessoal e académico, nomeadamente na perceção de autodomínio do estudante sobre a vida (Duffy et al., 2015; Hirschi, 2009; Konstam et al., 2015; Minkov, 2009; Rudolph et al., 2017). Os dados perspetivam Controlo como uma das variáveis intrinsecamente associadas aos objetivos do papel de estudante, pelo menos nesta fase de vida, que se traduz em autonomia, florescimento e confronto com desafios constantes de desenvolvimento e progressão. Neste sentido, os contextos institucionais devem criar condições de educabilidade que favoreçam a autodeterminação, quer no desenho de currículos quer em atividades extracurriculares formativas de competência e desenvolvimento. De salientar que Controlo tem correlação negativa (.34) com Ansiedade à Avaliação, sendo este dado corroborado pela relação negativa entre adaptabilidade e experiências de stress (e.g., Konstam et al., 2015; Maggiori et al., 2013; Merino-Tejedor et al., 2016; Rudolph et al., 2017). Estes dados têm implicações para o bem-estar geral mas também para a aprendizagem e saúde, posicionando a intervenção educacional numa abordagem holística do estudante e sobretudo da sua relação com o ambiente académico.

Neste estudo, os resultados de Confiança são ainda explicativos de Bem-estar e a respetiva correlação é de .54. Este dado é comprovado por trabalhos anteriores que demonstram a crença de capacidade pessoal como fator significativo nas escolhas e implementação de um projeto de vida (e.g., Savickas \& Porfeli, 2012) e dados que mostram autoeficácia geral como explicativa de bem-estar em estudantes do ensino superior (Teixeira \& Costa, 2017). A Confiança, considerada como o conjunto de crenças para lidar com as tarefas de carreira, é uma variável relevante, que implica uma avaliação de competência e perspetiva de sucesso nos vários desafios e tarefas. Os dados que relacionam Bem-estar com Controlo e Confiança convergem na importância atribuída aos recursos internos na resiliência e adaptação ao longo do desenvolvimento (Savickas \& Porfeli, 2012) e propõem uma reflexão sobre a necessidade da organizar, desde a infância, padrões educacionais que favoreçam experiências de autonomia, autorregulação, confiança e flexibilidade psicológica.

No entanto, sublinha-se que Curiosidade e Preocupação pouco contribuem para explicar Bem-estar, o que pode significar que estes recursos não são mobilizados em simultâneo para lidar com tarefas no percurso de carreira (Duffy et al., 2015; Hirschi, 2009; Maggiori et al., 2013; Rudolph et al., 2017). Nos estudantes dos dois primeiros anos da universidade, este dado pode estar associado ao facto de que a "grande" decisão foi tomada há relativamente pouco tempo, não existindo premência no acionamento destes recursos, nesta fase de vida. Apesar da adaptabilidade de carreira, no seu todo, explicar significativamente o bem-estar, o peso diferente de cada dimensão 
propõe um olhar construtivista de carreira, com diferentes necessidades de desenvolvimento pessoal nos diferentes tempos de vida e na diversidade dos contextos.

Os dados da regressão múltipla linear evidenciam a influência da Perspetiva de Carreira, no Bem-estar, confirmando $\mathrm{H} 2$. Esta dimensão inclui sentimentos de congruência, perspetivas de empregabilidade e prestígio sobre o curso. De referir que Perspetiva de Carreira demonstra correlações positivas e significativas com os quatro recursos de Adaptabilidade de Carreira e ainda com Bem-estar. Estes dados validam que esta dimensão pertence ao domínio dos recursos de carreira e são corroborados pelos trabalhos que demonstram a importância da congruência e do ajustamento ao curso no Bem-estar, em estudantes do ensino superior (Lent \& Brown, 2013; Teixeira \& Costa, 2017). Estes dados são ainda associados ao papel atribuído à contínua persecução dos objetivos nos níveis de bem-estar subjetivo (e.g., Lent et al., 2009), sendo o ensino superior terreno propício ao florescimento de potencialidades e de atualização de objetivos, principalmente se as decisões forem tomadas com autonomia pelo próprio estudante. Neste sentido, os dados legitimam ainda a relação da carreira com bem-estar, nomeadamente no papel da adaptabilidade de carreira no florescimento de capacidades, no desenvolvimento da identidade e no ajuste de crenças e expetativas.

Nas variáveis do domínio social, os resultados da regressão múltipla linear mostram o fator Relação com Pares como preditor de Bem-estar, confirmando H3. Estes dados são comprovados pelo papel demonstrado pelos recursos sociais na adaptação, tal como proposto pelos modelos de impacto (Astin \& Sax, 1998; Pascarella \& Terenzini, 1980). Nesta área, outros trabalhos têm mostrado o efeito da perceção da relação social nos comportamentos adaptativos no Ensino Superior (Diener \& Ryan, 2009; Lent, et al., 2009; Ryff \& Keyes, 1995). Estes dados colocam a questão sobre o impacto das relações sociais no rendimento académico, que ainda tem muito peso na conceção de sucesso, essencialmente se for concebido em termos de investimento pessoal e tempo despendido. Neste caso, salientam-se as iniciativas de estruturas institucionais, como os gabinetes de apoio ou associações académicas, bem como as atividades extracurriculares, que facilitam aproximação interpessoal e desenvolvimento de projetos comuns. Neste sentido, ainda se realça a importância do ambiente relacional dentro e fora da sala de aula, entre docentes e discentes e a comunidade no geral, quer na aplicação de práticas pedagógicas cooperativas e participativas quer no clima relacional de disponibilidade e ajuda.

Nas variáveis do domínio académico, é ainda preditor de Bem-estar a competência de Gestão do Tempo, sendo preditor negativo a Ansiedade à Avaliação, confirmando parcialmente H4. A relação entre Gestão de Tempo e Bem-estar pode também encontrar confirmação nos modelos de impacto, que propõem a relação entre envolvimento, competências académicas e adaptação, sendo assim importante o treino de metacognição e autorregulação (e.g., Costabile et al., 2013; Zimmerman, 2002). Estes dados sugerem o papel chave da gestão pessoal na regulação do comportamento, bem como no desenvolvimento e bem-estar. Neste sentido, salienta-se a importância da educabilidade desta competência, ao nível da intervenção com os estudantes desde a infância, e ao nível das intervenções com educadores, nomeadamente pais e professores.

A relação negativa entre Ansiedade à Avaliação e Bem-estar é corroborada pelas pesquisas que relacionam situações de stress, bem-estar e adaptação académica (e.g., Merino-Tejedor et al., 2016), expressando-se neste estudo nas correlações negativas de Ansiedade à Avaliação com Controlo e Bem-estar. A componente de ansiedade à avaliação sugere, por um lado, uma conceção reducionista de atribuir sucesso e "valor" pessoal ao rendimento académico, em notas ou médias (Soares et al., 2015; Soares et al., 2006), e, por outro lado, as dificuldades do estudante em lidar com situações de stress, que as avaliações envolvem. A associação entre sucesso e notas escolares aumenta a pressão dos estudantes e coloca a necessidade de a intervenção contemplar tanto a componente académica de métodos de estudo como a componente emocional de lidar com situações de stress, que podem conduzir a situações de burnout, à desvinculação académica, insucesso e abandono escolares. No entanto, nestes dados é importante recordar que, em alguns casos, os dados foram observados em época de exames.

Os dados da estatística inferencial revelam efeitos do ano curricular, confirmando parcialmente H5. Os estudantes do $2^{\circ}$ ano revelam níveis superiores em Controlo, Curiosidade, Confiança e Estudo e Aprendizagem, e inferiores em Ansiedade à Avaliação, comparativamente aos do $1^{\circ}$ ano. Este indicador pode sugerir uma capacidade ou tendência natural de adaptação que pode ser influenciada pela persistência, frequência e qualidade de sucessos, relações desenvolvidas e histórico individual, bem como pelo suporte institucional recebido pelos estudantes durante o primeiro ano. Apesar da metodologia transversal usada, estes dados sugerem que o processo de adaptação é contínuo e multidimensional, em que entram variáveis de natureza social, vocacional e académica, entre outras (e.g., Chickering \& Schlossberg, 1995; Soares et al., 2015). O conjunto dos dados salienta ainda a importância da intervenção nos primeiros tempos do ensino superior, que 
se traduz também por desenvolvimento pessoal ao longo da vida. Os estudos longitudinais serão clarificadores dos fatores que entram no processo de adaptação e sucesso no ensino superior, por proporcionarem dados ao longo do percurso académico, principalmente nas fases de transição, em que são acionadas estratégias e competências específicas.

\section{Limitações}

Este trabalho tem limitações metodológicas, nomeadamente: 1. o design experimental, que envolveu um único momento de recolha de dados. A metodologia longitudinal é mais adequada para analisar as variáveis responsáveis pelos processos de transição e adaptação, pois permite uma visão da evolução dos estudantes ao longo dos primeiros anos; 2 . a recolha de dados foi realizada próximo da época de exames, podendo influenciar os resultados, nomeadamente da Ansiedade à Avaliação; 3. características da amostra da sobre representação de raparigas e de estudantes de Psicologia, que são uma limitação comum neste tipo de pesquisa mas que podem influenciar o estudo das variáveis ligadas às dificuldades no ensino superior; 4. a utilização de instrumentos de autorrelato em dimensões significativas da conceção pessoal como estudante ou pessoa em desenvolvimento, pode incluir efeitos de desejabilidade social e aquiescência; e 5. a limitação em termos variáveis incluídas, já que no bem-estar e no sucesso podem ter influencia direta ou indireta variáveis como traços de personalidade, crenças de autoeficácia académica, atividades extracurriculares e de suporte social e académico.

\section{Conclusões}

Apesar das limitações apontadas, este estudo tem natureza inovadora por integrar variáveis de natureza demográfica, social, académica e vocacional e clarificar alguns dos fatores que entram no sistema autorregulatório de gestão da carreira no processo de adaptação ao ensino superior. A análise destes fatores contribui, por um lado, para aprofundar os quadros teóricos explicativos das transições e do sucesso e bem-estar no ensino superior (e.g., Lent et al., 2009), e, por outro, facultar indicadores aos responsáveis pelas políticas educativas e aos profissionais que atuam nas instituições. Na perspetiva de Lent e Brown (2013), estes fatores atuam em conjunto e as competências de adaptação podem ser aprendidas, tal como estratégias de bem-estar.

Na reflexão que estes dados proporcionam, é significativo que as intervenções se fundamentem num quadro multidimensional de natureza académica, pessoal, social e de carreira, potencializando as competências dos estudantes na adaptação e sucesso. As instituições de ensino superior poderão ter um papel relevante em promover a integração social entre pares mas também na adequação dos meios pedagógicos e curriculares de desenvolvimento de competências como gestão de tempo ou de redução de stress e ansiedade nas situações de avaliação. É ainda fator para reflexão o papel das dimensões de carreira no sucesso e bem-estar dos estudantes, ao nível das competências de adaptabilidade, perceção das possibilidades individuais e ao nível da empregabilidade e de reconhecimento social das próprias instituições. Estes dados impõem que as instituições do ensino superior concentrem esforços em criar condições para que os estudantes possam gerir a sua carreira, potenciando o funcionamento positivo e a satisfação com a vida. Neste âmbito, é também imprescindível a criação de redes sociais de suporte à empregabilidade e à inserção profissional.

É essencial sublinhar que as intervenções com tempo e estrutura preparam os estudantes para as transições, com efeitos na adaptação, sucesso e bem-estar do aluno e da comunidade escolar. Os efeitos destas ações revertem para o estudante, mas também para as próprias instituições do ensino superior, com repercussões na qualidade do ensino superior e na economia do país.

\section{Referências}

Almeida, L. S., Araújo A. M., \& Martins, C. (2016). Transição e adaptação dos estudantes do $1^{\circ}$ ano: variáveis intervenientes e medidas de atuação. In L. S. Almeida, \& R. V. Castro (Orgs.), Ser Estudante no Ensino Superior: o caso dos estudantes do $1^{\circ}$ ano (pp. 146-164). Braga: Universidade do Minho, Centro de Investigação em Educação. Astin, A. W., \& Sax, L. J. (1998). How undergraduates are affected by service participation. Journal of College Student Development, 39(3), 251-263.

Bandura, A. (1997). Self-efficacy: The exercise of control. New York: Freeman.

Briggs, A. R. J., Clark, J., \& Hall, I. (2012). Building bridges: Understanding student transition to university. Quality in Higher Education, 18(1), 3-21. doi: 10.1080/13538322.2011.614468

Chickering, A. W., \& Schlossberg, N. K. (1995). How to get the most out of college. Boston: Allyn \& Bacon. 
Costabile, A., Cornoldi, C., De Beni, R. D., Manfredi, P., \& Figliuzzi, S. (2013). Metacognitive components of student's difficulties in the first year of university. International Journal of Higher Education, 2(4), 165-171.

Diener, E. \& Ryan, K. (2009). Subjective Well-being: A general overview. Journal of Psychology, 39(4), 391-406.

Diener, E., Wirtz, D., Tov, W., Kim-Prieto, C., Choi, D., Oishi, S., \& Biswas-Diener, R. (2009). New measures of wellbeing: Flourishing and positive and negative feelings. Social Indicators Research, 39, 247-266.

Direção-Geral de Estatísticas da Educação e Ciência (2015). Indicadores de transferência e de abandono no ensino superior português. Retirado de http://www.dgeec.mec.pt/np4/292/\%7B \$clientServletPath\%7D/?newsId=516\&file Name=Indicadores_transferencia_abandono_Ens.pdf

Duarte, M. E., Lassance, M. C., Savickas, M. L., Nota, L., Rossier, J., Dauwalder, J., Guichard, J., Soresi, S., Van Esbroeck, R., \& Vianen, A. (2015). A construção da vida: Um novo paradigma para entender a carreira no século XXI. Revista Interamericana de Psicología, 44(2), 392-406.

Duarte, M., E., Soares, M., Fraga, S., Rafael, M., Lima, M., Paredes, I., Agostinho, R., \& Djalo, A. (2012). Career adapt abilities scale-Portugal form: Psychometric properties and relationships to employment status. Journal of Vocational Behavior, 80(3), 725 729.doi: 10.1016/j.jvb.2012.01.019

Duffy, R. D., Douglass, R. P., \& Autin, K. L. (2015). Career adaptability and academic satisfaction: Examining work volition and self-efficacy as mediators. Journal of Vocational Behavior, 90, 46-54.

Ferreira, F., \& Fernandes, P. (2015). Fatores que influenciam o abandono no ensino superior e iniciativas para a sua prevenção: O olhar de estudantes. Educação, Sociedade e Culturas, 45, 117-197.

Hirschi, A. (2009). Career adaptability development in adolescence: Multiple predictors and effect on sense of power and life satisfaction. Journal of Vocational Behavior, 74(2), 145-155. doi:10.1016/j.jvb.2009.01.002

Keyes, C. L. M. (2006). Subjective well-being in mental health and human development research worldwide: An introduction. Social Indicators Research, 77(1), 1-10.

Konstam, V., Celen-Demirtas, S., Tomek, S., \& Sweeney, K. (2015). Career adaptability and subjective well-being in unemployed emerging adults: A promising and cautionary tale. Journal of Career Development, 42(6),1-15.

Lent, R. W., \& Brown, S. D. (2013). Social cognitive model of career self-management: Toward a unifying view of adaptive career behavior across the life span. Journal of Counseling Psychology, 60(4), 557-568.

Lent, R. W., Taveira, M. C., Sheu, H., \& Singley, D. (2009). Social cognitive predictors of academic adjustment and life satisfaction in Portuguese college students: A longitudinal analysis. Journal of Vocational Behavior, 74, 190-198.

Maggiori, C., Johnston, C., Krings, F., Massoudi, K., \& Rossier, J. (2013). The role of career adaptability and work conditions on general and professional well-being. Journal of Vocational Behavior, 83, 437-449.

Merino-Tejedor, E., Hontangas, P. M., \& Boada-Grau, J. (2016). Career adaptability and its relation to self-regulation, career construction, and academic engagement among Spanish university students. Journal of Vocational Behavior, 93, 92-102.

Minkov, M. (2009). Predictors of differences in subjective well-being across 97 nations.Cross-Cultural Research, 43(2), 152-179.

Pascarella, E. T., \& Terenzini, P. T. (1980). Predicting freshman persistence and voluntary dropout decisions from a theoretical model. The Journal of Higher Education, 51(1), 60-75.

Robertson, P. J. (2015). Positive psychology: A movement to reintegrate career counselling within counselling psychology? Counselling Psychology Review, 30(3), 26-36.

Rudolph, C. W., Lavigne, K. N., Katz, I. M., \& Zacher, H. (2017). Linking dimensions of career adaptability to adaptation results: A meta-analysis. Journal of Vocational Behavior, 102, 151-173.

Ryff, C. D., \& Keyes, C. L. M. (1995). The structure of psychological well-being revisited. Journal of Personality and Social Psychology, 69, 719-727.

Savickas, M. L. (2013). Career construction theory and practice. In R. W. Lent \& S. D. Brown (Eds.), Career development and counseling: Putting theory and research to work (2nd ed) (pp. 144-180). Hoboken, NJ: John Wiley.

Savickas, M. L., \& Porfeli, E. J. (2012).Career Adapt-Abilities Scale: Construction, reliability, and measurement equivalence across 13 countries. Journal of Vocational Behavior, 80, 661-673.

Seligman, M. E. P. (2011). Flourish: A visionary new understanding of happiness and well-being (pp. 3-29). New York, NY: Free Press.

Siqueira, M. M. M., \& Padovam, V. A. R. (2008). Bases teóricas de bem-estar subjetivo, bem-estar psicológico e bemestar no trabalho. Psicologia: Teoria e Pesquisa, 24(2), 201-209. 
Soares, A., Almeida, L. S., \& Ferreira, J. (2006). Questionário de Vivências Académicas: Versão integral (QVA) e versão reduzida (QVA-r). In M. Goncalves, L. Almeida, \& M. Simões (Coords.), Avaliação psicológica: Instrumentos validados para a população portuguesa (2a ed. atualizada, Vol. 1, pp. 101-120). Coimbra: Quarteto.

Soares, A. M., Pinheiro, R. M., \& Canavarro, J. M. P. (2015). Transição e adaptação ao ensino superior e a demanda pelo sucesso nas instituições portuguesas. Psychologica, 58(2), 97-116. doi: http://dx.doi.org/10.14195/1647-8606_58 2_6

Steele, C., Andrews, H., \& Upton. D. (2012). Statistics in psychology. Harlow: Pearson Education.

Teixeira, M. A. P., Bardagi, M. P., Lassance, M. C. P., Magalhães, M. O., \& Duarte, M. E. (2012). Career Adapt-Abilities Scale - Brazilian Form: Psychometric properties and relationships to personality. Journal of Vocational Behavior, $80,680-685$.

Teixeira, M. O. \& Costa, C. J. (2017). Carreira e bem-estar subjetivo no ensino superior. Revista Brasileira de Orientação Profissional, 18(1), 19-29.

Zimmerman, B. J. (2002). Becoming a self-regulated learner: An overview. Theory Into Practice, 41(2), 64-70.

Recebido 15/02/2018

Reformulado 03/06/2018

Aceite Final 07/10/2018

Sobre os autores

Bruno Rocha Almeida é Mestre em Psicologia da Educação e da Orientação.

Maria Odília Teixeira é Professora Auxiliar da Faculdade de Psicologia da Universidade de Lisboa. Especialista no domínio do aconselhamento de carreira, com diversas publicações neste campo. 